\title{
The Actin-Binding Protein Canoe/AF-6 Forms a Complex with Robo and Is Required for Slit-Robo Signaling during Axon Pathfinding at the CNS Midline
}

\author{
Jana Slováková, ${ }^{1}$ Stephan Speicher, ${ }^{1}$ Natalia Sánchez-Soriano, ${ }^{2}$ Andreas Prokop, ${ }^{2}$ and Ana Carmena ${ }^{1}$ \\ ${ }^{1}$ Instituto de Neurociencias, CSIC/UMH, 03550 Sant Joan d'Alacant, Spain, and ${ }^{2}$ Faculty of Life Sciences, Wellcome Trust Centre for Cell-Matrix Research, \\ Manchester M13 9PT, United Kingdom
}

\begin{abstract}
Axon guidance is a key process during nervous system development and regeneration. One of the best established paradigms to study the mechanisms underlying this process is the axon decision of whether or not to cross the midline in the Drosophila CNS. An essential regulator of that decision is the well conserved Slit-Robo signaling pathway. Slit guidance cues act through Robo receptors to repel axons from the midline. Despite good progress in our knowledge about these proteins, the intracellular mechanisms associated with Robo function remain poorly defined. In this work, we found that the scaffolding protein Canoe (Cno), the Drosophila orthologue of AF-6/Afadin, is essential for Slit-Robo signaling. Cno is expressed along longitudinal axonal pioneer tracts, and longitudinal Robo/Fasciclin2-positive axons aberrantly cross the midline in cno mutant embryos. cno mutant primary neurons show a significant reduction of Robo localized in growth cone filopodia and Cno forms a complex with Robo in vivo. Moreover, the commissureless (comm) phenotype (i.e., lack of commissures due to constitutive surface presentation of Robo in all neurons) is suppressed in comm, cno double-mutant embryos. Specific genetic interactions between $c n o$, slit, robo, and genes encoding other components of the Robo pathway, such as Neurexin-IV, Syndecan, and Rac GTPases, further confirm that Cno functionally interacts with the Slit-Robo pathway. Our data argue that Cno is a novel regulator of the Slit-Robo signaling pathway, crucial for regulating the subcellular localization of Robo and for transducing its signaling to the actin cytoskeleton during axon guidance at the midline.
\end{abstract}

\section{Introduction}

The midline of the CNS is a crucial boundary linking the two symmetric neurogenic regions of bilateral organisms. Commissural axons cross the midline to the contralateral side and never recross, whereas other axons stay on the ipsilateral side. These decisions depend on evolutionary conserved axon guidance cues provided by specialized midline cells, such as the floorplate in the vertebrate spinal cord or midline glia cells in the ventral nerve cord of Drosophila (Colamarino and Tessier-Lavigne, 1995; Bashaw and Klein, 2010; Evans and Bashaw, 2010). The secreted protein Slit (Sli) belongs to these guidance molecules critical for the decision of whether or not to cross the midline, acting through its receptor Roundabout (Robo) to repel axons from the

\footnotetext{
Received Dec. 20, 2011; revised May 7, 2012; accepted May 29, 2012.

Author contributions: A.C. designed research; J.S. and S.S. performed research; J.S., N.S.-S., and A.C. analyzed data; A.P. and A.C. wrote the paper.

This work was supported by grants from the British Biotechnology and Biological Sciences Research Council (BB/I002448/1) to A.P. and from the Spanish government (BFU2006-09130, BFU2009-08833, and CONSOLIDERINGENI0 2010 (SD2007-00023) to A.C. The Fly Facility in Manchester is supported by funds from The University of Manchester and the Wellcome Trust (087742/Z/08/Z). We thank Greg Bashaw, Guy Tear, the Bloomington Drosophila Stock Center at Indiana University, and the Developmental Studies Hybridoma Bank at the University of lowa for kindly providing fly strains and antibodies. We also thank Raquel Pérez-Gómez for helping with the statistic analyses. J. S. holds a JAE (Junta de Ampliación de Estudios) predoctoral fellowship from the Spanish Research Council, cofinanced by the European Social Fund.

The authors declare no competing financial interests.

Correspondence should be addressed to Ana Carmena, Avda. Ramón y Cajal s/n, 03550-Sant Joan d'Alacant, Alicante, Spain. E-mail: acarmena@umh.es.

DOI:10.1523/JNEUROSCI.6342-11.2012

Copyright $\odot 2012$ the authors $\quad 0270-6474 / 12 / 3210035-10 \$ 15.00 / 0$
}

midline (Kidd et al., 1998, 1999; Brose et al., 1999; Ypsilanti et al., 2010). In Drosophila, Slit and Robo form a quaternary complex with Neurexin-IV (Nrx-IV) and the proteoglycan Syndecan (Sdc) (Johnson et al., 2004; Steigemann et al., 2004; Banerjee et al., 2010). A number of Robo downstream effectors have also been reported, such as the Abelson tyrosine kinase (Abl), the actin regulator Enabled (Ena), and the Rac-guanine nucleotide exchange factor (GEF) Son of sevenless (Sos) (Bashaw et al., 2000; Hakeda-Suzuki et al., 2002; Ng et al., 2002; Hsouna et al., 2003; Yang and Bashaw, 2006), but the mechanisms by which Slit-Robo signaling is regulated and transduced within growing axons are still poorly understood (Dickson and Gilestro, 2006; SánchezSoriano et al., 2007; O’Donnell et al., 2009).

The best known Robo regulator is the transmembrane protein Commissureless (Comm). Comm suppresses Robo function by binding it in the Golgi of contralateral projecting neurons and directing it for degradation, thus allowing commissural axons to cross the midline. Accordingly, in comm mutant embryos, Robo becomes enriched at growth cones of all neurons, which are repelled from the midline and fail to form any commissures.

Here we report a further regulator of Robo and antagonist of Comm, called Canoe (Cno). Cno and its vertebrate homologues AF-6/Afadin are PDZ (Postsynaptic density-95/Discs large/Zona occludens-1) domain-containing scaffolding proteins. They are predominant at adherens junctions (AJs) (Miyamoto et al., 1995), where they regulate the linkage of AJs to the actin cytoskeleton (Mandai et al., 1997; Takahashi et al., 1998; Matsuo et al., 


\section{Cno/Fas2}
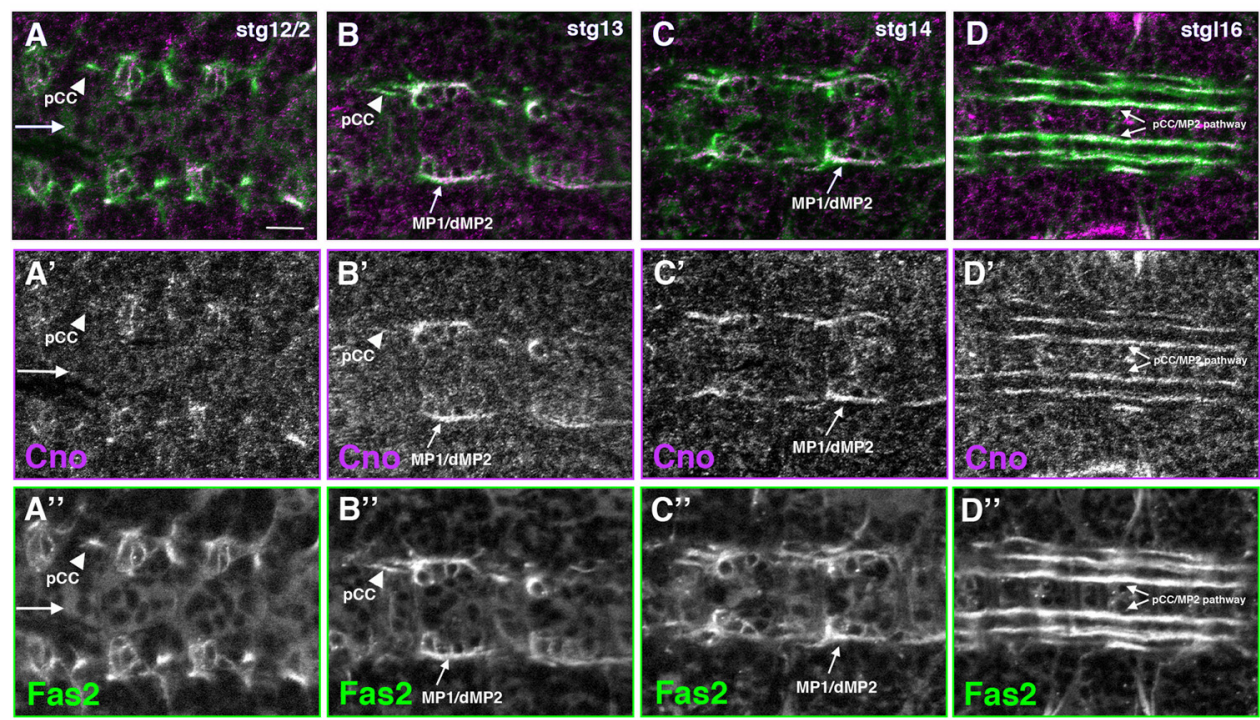

Cno/22C10
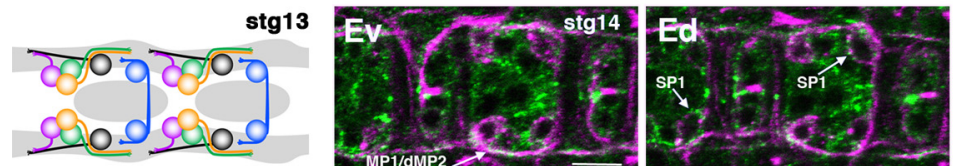

Cnoleagle-Gal4>>

UAS-CD 8::GFP
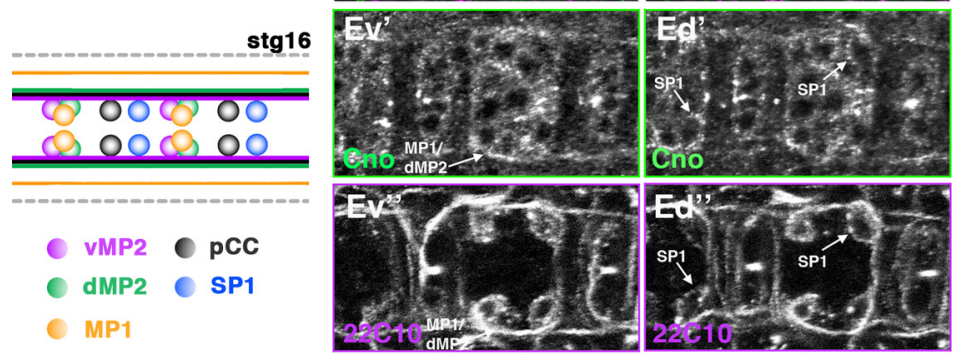

Fas2

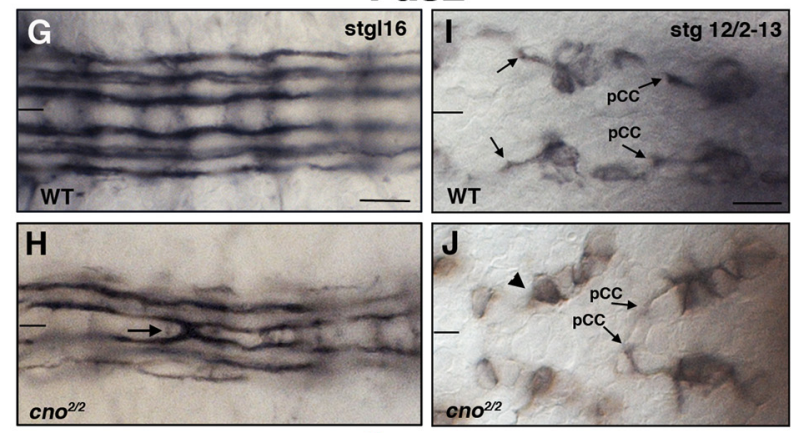

o
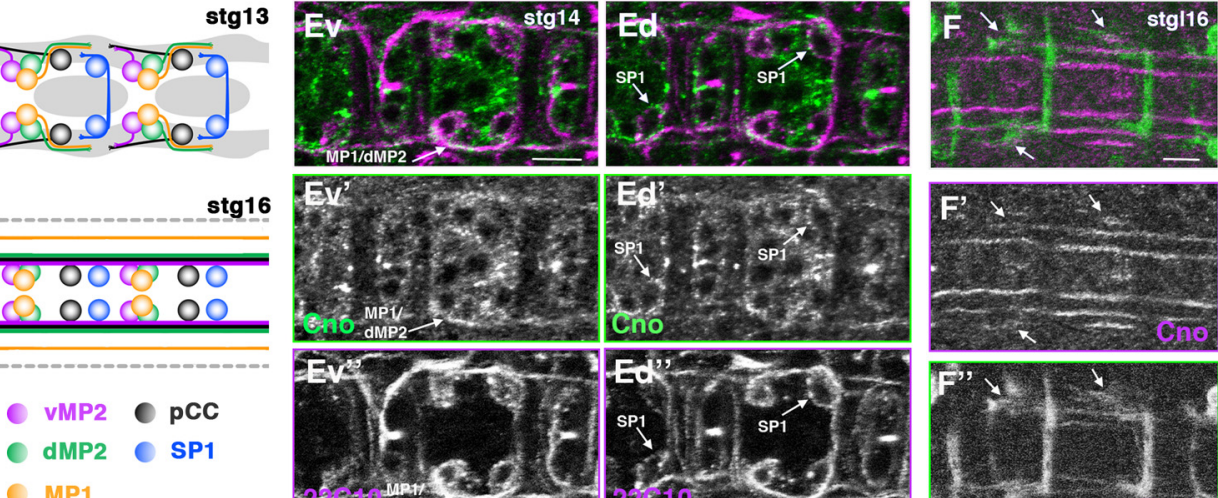

no
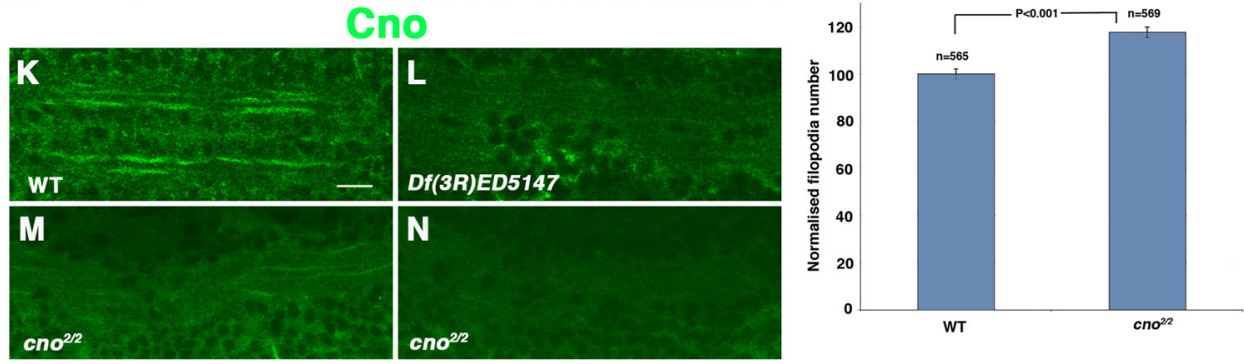

Figure 1. Cno is expressed along pioneer ipsilateral axons and is required during axon pathfinding. $\boldsymbol{A}-\boldsymbol{D}^{\prime \prime}$, Ventral views of Drosophila embryos stained with Cno (magenta) and Fas2 (green) throughout neuronal differentiation. Cno colocalizes with Fas2 in ipsilateral pioneer neurons since early stages (stg 12/2). Arrows (A-A') point to the midline. Cno is strongly detected in Fas2-positive axons, which include the ipsilateral pCC (A-B', arrowheads) and MP1/dMP2 neurons (B-C", arrows). Later, during stage 16, Cno is still detected along Fas2-positive longitudinal tracts, including the most medial pCC/MP2 pathway (D-D"', arrows). The schematic drawing represents the position of the indicated subset of neurons at stages 13 and 16 . Ev-Ed", Two different focal planes of the ventral cord, more ventral (v) and more dorsal (d) of a stage 14 Drosophila embryo stained with Cno (green) and 22C10 (magenta). In a ventral view (Ev-Ev'), (Figurelegend continues.) 
1999; Lorger and Moelling, 2006; Sawyer et al., 2009). Cno also performs AJ-independent functions regulating signal integration and asymmetric cell division (Carmena et al., 2006; Speicher et al., 2008; Slováková and Carmena, 2011). Here, we found that Cno is required for Robo signaling. Embryos lacking Cno function showed ectopic midline crossing of axons, and this phenotype was enhanced in sensitized genetic backgrounds in which the Slit-Robo pathway was impaired. Cno coimmunoprecipitated with Robo from embryos, and it promoted subcellular localization to growth cone filopodia of embryo-derived primary neurons. Notably, the comm phenotype was strikingly suppressed in comm, cno double mutants, indicating an essential Cno requirement for Robo signaling.

\section{Materials and Methods}

Drosophila strains and genetics. The following mutant stocks (males and females) were used [all from the Bloomington Stock Center (http:// flystocks.bio.indiana.edu/), unless another source specified]: $c n o^{2}$ (Jürgens et al., 1984); $c n o^{R 2}$ (Sawyer et al., 2009); Df(3R)ED5147 (deficiency uncovering the cno locus; Drosophila Genetic Resource Center, Kyoto Institute of Technology); $s i^{2}$ (Rothberg et al., 1988; Kidd et al., 1999); robo $^{2}$ (Seeger et al., 1993); Sos ${ }^{34 E a-6}$ (Rogge et al., 1991); $\mathrm{Mtl}^{\Delta}$, Racl ${ }^{111}$, and $\operatorname{Rac}^{\Delta}{ }^{\Delta}$ (Ng et al., 2002); Nrx-IV $V^{4304}$ (Baumgartner et al., 1996); $S d c^{97}$ (Steigemann et al., 2004); $\mathrm{comm}^{1}$ and $\mathrm{comm}^{5}$ (Tear et al., 1996); $A b l^{4}$ (Henkemeyer et al., 1988; Bashaw et al., 2000); ena ${ }^{G C 5}$ (Gertler et al., 1995; Bashaw et al., 2000); Nrx-IV-GFP (CA06597) (Morin et al., 2001); eagle-Gal4 (Ito et al., 1995); elav-Gal4 (Kolodziej, P., 2005.5, FlyBase); slit-Gal4 (Scholz et al., 1997); UAS-mCD8::GFP (Lee and Luo, 1999); UAS-robo::MYC (a gift from G. Bashaw); and UAS-cno::GFP (Slováková and Carmena, 2011). All the crosses GAL4-UAS ( $\sim 50$ females $\times 50$ males) were performed at $29^{\circ} \mathrm{C} . y w$ (males and females) was used as the reference control wild-type strain. Balancer chromosomes containing different lac $Z$ or GFP transgenes were used for identification of homozygous mutant embryos.

Immunohistochemistry, immunofluorescence, and microscopy. Embryo fixation and antibody staining were performed by standard protocols. The following primary antibodies were used: rabbit anti-Cno, 1/400-1/ 1000 (Speicher et al., 2008); mouse BP102 1/200, mouse anti-Robo $1 / 100,1 \mathrm{D} 4$ mouse anti-Fas2 $1 / 100$, and 22C10 mouse anti-Futsch $1 / 20$ (all from Developmental Studies Hybrydoma Bank); rabbit anti- $\beta$ galactosidase $1 / 100,000$ (Cappel). Secondary antibodies coupled to biotin (Vector Laboratories), Alexa 488, Alexa 546, or Alexa 633 (Invitrogen) were used. For immunostaining with the anti-Cno antibody, embryos were fixed by using the heat-methanol method (Tepass, 1996). The Zenon Mouse IgG Labeling Kit (Invitrogen) was used for doublestaining Fas2 and Robo. Images were recorded by using a Zeiss Axio Imager.A1 and a Zeiss AxioCam HRc. Fluorescent images were recorded by using a Leica upright DM-SL confocal microscope and assembled by using Adobe Photoshop.

Coimmunoprecipitations. For in vivo coimmunoprecipitations, lysates were prepared from $\sim 200 \mu \mathrm{l}$ of 16-18 h elav-Gal4 $\gg U A S-c n o:: G F P$,

\footnotetext{
(Figure legend continued.) Cno is detected along with $22 \mathrm{C} 10$ in the ipsilateral MP1/dMP2 neurons (arrows). In a more dorsal view (Ed-Ed'), low levels of Cno are present in the cell body of the SP1 contralateral neurons (arrows). $\boldsymbol{F}-\boldsymbol{F}^{\prime \prime}$, At late stage 16 , (no is observed in contralateral Eagle ${ }^{+}$axons that have already crossed the midline (arrows). $\mathbf{G}-\boldsymbol{H}$, In WT late stage 16 embryos, Fas2 is expressed in three longitudinal fascicles at each side of the midline $(\boldsymbol{G})$. In $\mathrm{CnO}^{2 / 2}$ mutants, Fas2-positive axons cross the midline ( $\boldsymbol{H}$, arrow; see also Fig. 2G). $\boldsymbol{I}, \boldsymbol{J}$, At early stages (12/2-13), guidance defects in Fas2-positive pioneer neurons, such as pCC (arrows), are already detected in $\mathrm{Cn}^{2 / 2}$ mutants. Defects in axon outgrowth are also observed (, , arrowhead). $\boldsymbol{K}-\boldsymbol{N}$, In WT embryos, Cno is clearly detected in longitudinal axon bundles $(\boldsymbol{K})$, whereas this expression steeply drops or disappears in Df(3R)ED5147 ( $(\mathbf{L})$ and $c n 0^{2 / 2}(\boldsymbol{M}, \boldsymbol{N})$ homozygous mutant embryos. $\boldsymbol{O}$, In $\mathrm{CnO}^{2 / 2}$ primary neuron cultures, the number of filopodia per neuron was significantly increased compared with WT neurons ( $n=$ number of neurons analyzed). Tubulin (Tub) is in green and F-actin (Phalloidin) is in magenta. Scale bars: $\mathbf{A}-\boldsymbol{N}, 10$ $\mu \mathrm{m} ; 0,5 \mu \mathrm{m}$.
}

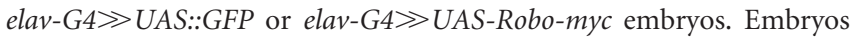
were homogenized in lysis buffer [ $50 \mathrm{~mm}$ Tris, $\mathrm{pH} 8,150 \mathrm{~mm} \mathrm{NaCl}, 0.1 \%$ SDS, 1 mM EDTA, 1\% Triton X-100, 1 mm NaF, $100 \mu \mathrm{M} \mathrm{Na} \mathrm{VO}_{4}, 2 \mathrm{~mm}$ PMSF, and Complete Protease Inhibitors (Roche)]. Extracts were centrifuged first for $15 \mathrm{~min}$ at $14,000 \mathrm{rpm}(18,700 \mathrm{~g})$ at $4^{\circ} \mathrm{C}$ and then $5 \mathrm{~min}$ under the same conditions. The cleared extract was incubated with rabbit polyclonal antibody to GFP Sepharose beads (Abcam) for $2 \mathrm{~h}$ at $4^{\circ} \mathrm{C}$. The elav-G4 $\gg U A S-R o b o-m y c$ embryo extract was precleared with Protein $\mathrm{G}$ beads for $2 \mathrm{~h}$ at $4^{\circ} \mathrm{C}$ followed by incubation with mouse anti-c-Myc (BD) or with a mouse serum (as a negative control) overnight at $4^{\circ} \mathrm{C}$. The supernatant-antibody mix was incubated with $40-60 \mu$ l of prewashed Protein $\mathrm{G}$ beads for $2 \mathrm{~h}$ at $4^{\circ} \mathrm{C}$. The beads then were washed three times with lysis buffer without inhibitors, resuspended in protein-set buffer (Fluka), and heated at $95^{\circ} \mathrm{C}$ for $3 \mathrm{~min}$. Precipitates were resolved by SDS-PAGE and immunoblotted with mouse anti-GFP (Clontech), rabbit anti-Cno (affinity purified) (Slováková and Carmena, 2011), mouse anti-c-Myc (BD) and anti-Robo (13C9, Developmental Studies Hybrydoma Bank). Each experiment was repeated at least two or three times.

Westerns and quantifications. Homozygote stage 16 mutant embryos $\left(\mathrm{cno}^{2 / 2}\right.$ or $\left.\mathrm{comm}^{5 / 5}\right)$ were selected by the lack of GFP. Embryonic extracts were treated as described above. The intensity of the protein bands was measured with Fujifilm LAS-1000 equipment and quantified using Quantity One 4.6 software (Bio-Rad). A nonparametric rank-based analysis using N-PAR1WAY procedure with SAS system v9.2 statistics package was applied, as the data obtained did not follow a normal distribution. Protein levels are expressed as fold change over control upon normalization for the lowest WT protein/tubulin value $(n=2-3$ per each genotype).

Generation of Drosophila primary neuron cultures. Drosophila primary neuron cultures were generated as described previously (Prokop, 2012). In brief, cells were removed with micromanipulator-attached capillaries from stage 11 embryos $\left(6-7 \mathrm{~h}\right.$ after egg laying at $25^{\circ} \mathrm{C}$ ) (Campos-Ortega and Hartenstein, 1997), treated for $5 \mathrm{~min}$ at $37^{\circ} \mathrm{C}$ with dispersion medium, washed, and dissolved in $30-40 \mathrm{ml}$ of Schneider medium (Schneider, 1964). Then, the aliquots were transferred to coverslips and kept as hanging drop cultures in airtight special culture chambers, usually for $6 \mathrm{~h}$ at $26^{\circ} \mathrm{C}$.

Immunocytochemistry of neuron cultures. Cultured Drosophila neurons were analyzed $6 \mathrm{~h}$ after plating. They were fixed $(30 \mathrm{~min}$ in $4 \%$ paraformaldehyde- $0.05 \mathrm{~m}$ phosphate buffer, $\mathrm{pH} 7.2$ ), then washed in PBS $0.1 \%$ Triton X-100 (PBT). Incubation with antibodies was performed in PBT. Microtubules were stained with anti-tubulin (1:1000; SigmaAldrich) and FITC or Cy3-conjugated secondary antibodies (1:200; Jackson ImmunoResearch). Filamentous actin was detected with TRITCconjugated phalloidin (Sigma-Aldrich). In some experiments, to determine only surface staining of the protein, Robo staining (DSHB, 1:10) was performed in PBS without blocking reagents. Stained Drosophila neurons were mounted in Vecta-shield (Vector Laboratories).

Quantitations and statistic analyses. Primary neuron culture images were taken using an AxioCam camera mounted on an Olympus BX50WI microscope. Filopodia were identified as needle-like, phalloidin-stained surface protrusions; filopodia numbers reflect the total amount of filopodia per neuron. Statistic analyses were performed with Sigma-Aldrich Stat software using a $t$ test or Mann-Whitney rank sum test.

\section{Results \\ Cno is expressed in ipsilateral pioneer axonal tracts of the ventral nerve cord}

We had observed that Cno was present in the CNS during axon outgrowth (J. Slováková and A. Carmena, unpublished observations). In an attempt to characterize a potential function of Cno during this process, we analyzed that expression in detail. We performed double labeling of Cno and some well established specific markers, such as Fasciclin 2 (Fas2), which is expressed early in $\mathrm{pCC} / \mathrm{MP} 1 / \mathrm{MP} 2$ pioneer neurons that project ipsilaterally (Fig. 1, schematic diagram); mAb 22C10/Futsch, which localizes to a subset of well characterized neurons, including the contralateral SP1 neuron; and mCD8::GFP induced by the eagle-Gal4 driver 

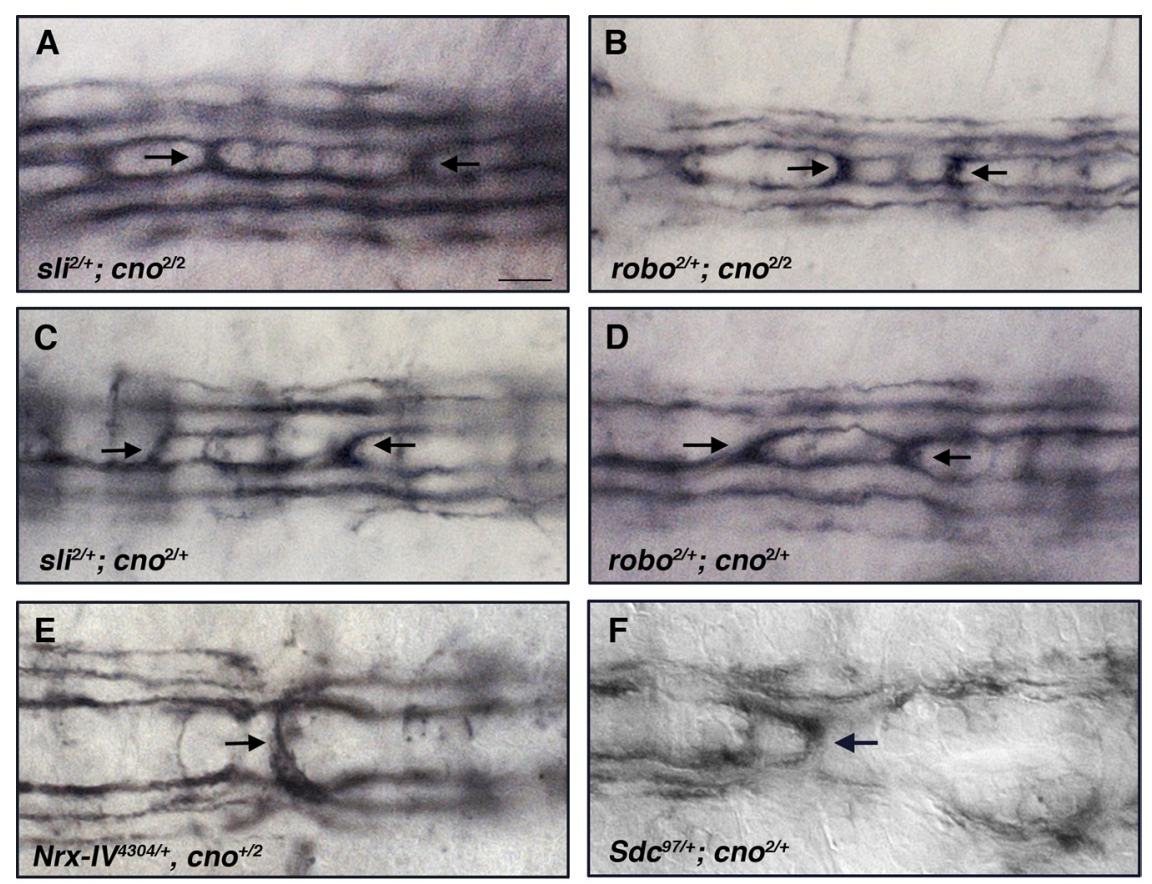

\section{G}

Genetic interactions between cno and upstream components of the Slit/Robo signaling pathway

\begin{tabular}{|c|c|c|c|c|c|c|}
\hline \multirow[b]{2}{*}{ Genotype } & \multicolumn{4}{|c|}{ axonal phenotypes } & \multirow[b]{2}{*}{$\begin{array}{l}\text { Total \# } \\
\text { embryos }\end{array}$} & \multirow[b]{2}{*}{$\begin{array}{l}\text { Total }(\% \\
\text { embryos } \\
\text { with P } \\
\end{array}$} \\
\hline & $\begin{array}{c}\text { Fasciculation } \\
(\%)\end{array}$ & $\begin{array}{c}\text { Outgrowth } \\
\qquad \%)\end{array}$ & $\begin{array}{l}\text { Guida } \\
\text { mild } \\
(\%)\end{array}$ & $\begin{array}{c}\text { ance* }^{*} \\
\text { severe } \\
(\%)\end{array}$ & & \\
\hline $\mathrm{Cno}^{2 / 2}$ & $\begin{array}{c}45 \\
(54 / 120)\end{array}$ & $\begin{array}{c}4.2 \\
(5 / 120)\end{array}$ & $\begin{array}{c}\mathbf{2 8 . 3} \\
(34 / 120)\end{array}$ & $\begin{array}{c}4.2 \\
(5 / 120)\end{array}$ & 120 & 58.3 \\
\hline$s l i t^{2 /+}$ & o & 0 & $\mathbf{0}$ & $\begin{array}{c}7.1 \\
(5 / 70)\end{array}$ & 70 & 7.1 \\
\hline$s l i t^{2 /+} ; \mathrm{cno}^{2 / 2}$ & $\begin{array}{c}38.4 \\
(43 / 112)\end{array}$ & $\begin{array}{c}9.8 \\
(11 / 112)\end{array}$ & $\begin{array}{l}\mathbf{5 . 4} \\
(6 / 112)\end{array}$ & $\begin{array}{l}\mathbf{5 8} \\
(65 / 112)\end{array}$ & 112 & 70.5 \\
\hline robo $^{2 /+}$ & o & 0 & $\mathbf{0}$ & $\begin{array}{c}1.1 \\
(1 / 90)\end{array}$ & 90 & 1.1 \\
\hline $\mathrm{robo}^{2 /+} ; \mathrm{Cno}^{2 / 2}$ & $\begin{array}{c}\mathbf{3 3 . 3} \\
(37 / 111)\end{array}$ & $\begin{array}{l}\mathbf{1 1 . 7} \\
(13 / 111)\end{array}$ & $\begin{array}{c}9.9 \\
(11 / 111)\end{array}$ & $\begin{array}{c}30.6 \\
(34 / 111)\end{array}$ & 111 & 65.7 \\
\hline $\mathrm{cno}^{2 /+}$ & $\begin{array}{l}\mathbf{2 . 5} \\
(1 / 40)\end{array}$ & $\mathbf{0}$ & 0 & $\mathbf{0}$ & 40 & 2.4 \\
\hline slit $^{2 /+} ;$ cno $^{2 /+}$ & $\begin{array}{c}16.7 \\
(11 / 66)\end{array}$ & 0 & $\mathbf{0}$ & $\begin{array}{l}\mathbf{1 9 . 7} \\
(13 / 66)\end{array}$ & 66 & 31.8 \\
\hline $\mathrm{robo}^{2 /+} ; \mathrm{cno}^{2 /+}$ & $\begin{array}{l}\mathbf{2 . 7} \\
(2,73)\end{array}$ & $\begin{array}{l}8.2 \\
(6 / 73)\end{array}$ & $\mathbf{0}$ & $\begin{array}{c}\underset{(17 / 73)}{23.3} \\
\text { (17/3) }\end{array}$ & 73 & 24.6 \\
\hline$N r x-I V^{4304 /+}, \mathrm{cno}^{+/ 2}$ & $\begin{array}{l}\mathbf{1 5 . 6} \\
(5 / 32)\end{array}$ & $\begin{array}{l}18.8 \\
(6 / 32)\end{array}$ & $\begin{array}{l}\mathbf{6 . 3} \\
(2 / 32)\end{array}$ & $\begin{array}{l}\mathbf{2 1 . 9} \\
(7 / 32)\end{array}$ & 32 & 40.6 \\
\hline$N r x-I V^{4.304 /+}$ & $\mathbf{0}$ & $\begin{array}{l}2.4 \\
(1 / 42)\end{array}$ & $\mathbf{0}$ & $\begin{array}{l}\mathbf{2 . 4} \\
(1 / 42)\end{array}$ & 42 & 4.8 \\
\hline$S d c^{97 /+} ; \mathrm{cno}^{2 /+}$ & $\mathbf{0}$ & $\begin{array}{l}9.3 \\
(4 / 43)\end{array}$ & $\begin{array}{l}4.7 \\
(2 / 43)\end{array}$ & $\begin{array}{l}11.6 \\
(5 / 43)\end{array}$ & 43 & 16.3 \\
\hline$S d c^{97 /+}$ & o & 0 & $\mathbf{0}$ & $\mathbf{0}$ & 25 & 0 \\
\hline $\begin{array}{l}\text { Midline guidance phe } \\
\text { connectives; severe def } \\
\mathrm{P}=\text { phenotype }\end{array}$ & $\begin{array}{l}\text { types: mild defec } \\
\text { ts include midlin }\end{array}$ & $\begin{array}{l}\text { include mispla } \\
\text { crossovers. }\end{array}$ & ement of & axon bund & dles within lc & ngitudinal \\
\hline
\end{tabular}

Figure 2. Cno functionally interacts with upstream components of the Slit-Robo signaling pathway during axon pathfinding $\boldsymbol{A}-\boldsymbol{F}$, Ventral views of stage 16 mutant embryos stained with anti-Fas2. Fas2 axons that normally extend longitudinally (see Fig. line, which is expressed in a subset of neurons, mostly projecting contralaterally (Grenningloh et al., 1991; Dittrich et al., 1997; Hidalgo and Brand, 1997; Schmid et al., 1999; Hummel et al., 2000). At early stages of neuronal differentiation (stage 12/2-14), Cno/Fas2 double staining revealed the presence of Cno along Fas2ipsilateral pioneer axonal tracts, such as pCC and MP1/dMP2 (Klämbt et al., 1991; Campos-Ortega and Hartenstein, 1997; Hidalgo and Brand, 1997) (Fig. 1 A$\left.C^{\prime \prime}\right)$. At later stages (late stage 16), Cno localized to the three longitudinal Fas2positive axon fascicles on each side of the midline, including the most medial pCC/MP2 pathway (Fig. 1 $D-D$ "). These longitudinal tracts include at this point not only ipsilateral neurons but also contralateral neurons that have already crossed the midline and joined these tracts on the other side. At this late stage, Cno was detected in some eagle-Gal4-positive axons, most of which are contralateral (Schmid et al., 1999) (Fig. 1F-F”). Double staining with $\mathrm{mAb} 22 \mathrm{C} 10$ in stage 12/2-14 embryos revealed that Cno was present at low levels in the cell body of SP1 contralateral neurons but not in the axons of these neurons (Fig. $1 E d-E d$ ”). Furthermore, staining confirmed the expression of Cno in ipsilateral axons of the MP1/dMP2 neurons known to be 22C10-positive (Fig. $1 E v-E v$ "). Thus, Cno is expressed during early stages of neuronal differentiation in ipsilateral axons when they decide not to cross the midline. At later stages, Cno seems to be upregulated in contralateral neurons, once they have crossed the midline.

\section{Cno is required for proper axon pathfinding at the midline}

Given that Cno is present in pioneer neurons from early stages of axon outgrowth, we wondered whether Cno was necessary during axon pathfinding. To address this point, we studied the $c n o^{2}$ loss-of-function mutant allele, which we used in our previous studies (Carmena et al., 2006; Speicher et al., 2008; Slováková and Carmena, 2011). As explained above, Fas2 labels three longitudinal axon fascicles on each side of the ventral nerve cord that never cross the midline in WT embryos (Hidalgo and Brand, 1997) (Fig.

$$
\leftarrow
$$

1G) cross the midline in the mutant condition indicated in each panel (arrows). G, Quantitations of genetic interactions between $\mathrm{Cno}$ and upstream components of the Slit-Robo pathway. Scale bar, $10 \mu \mathrm{m}$. 


\section{Fas2}
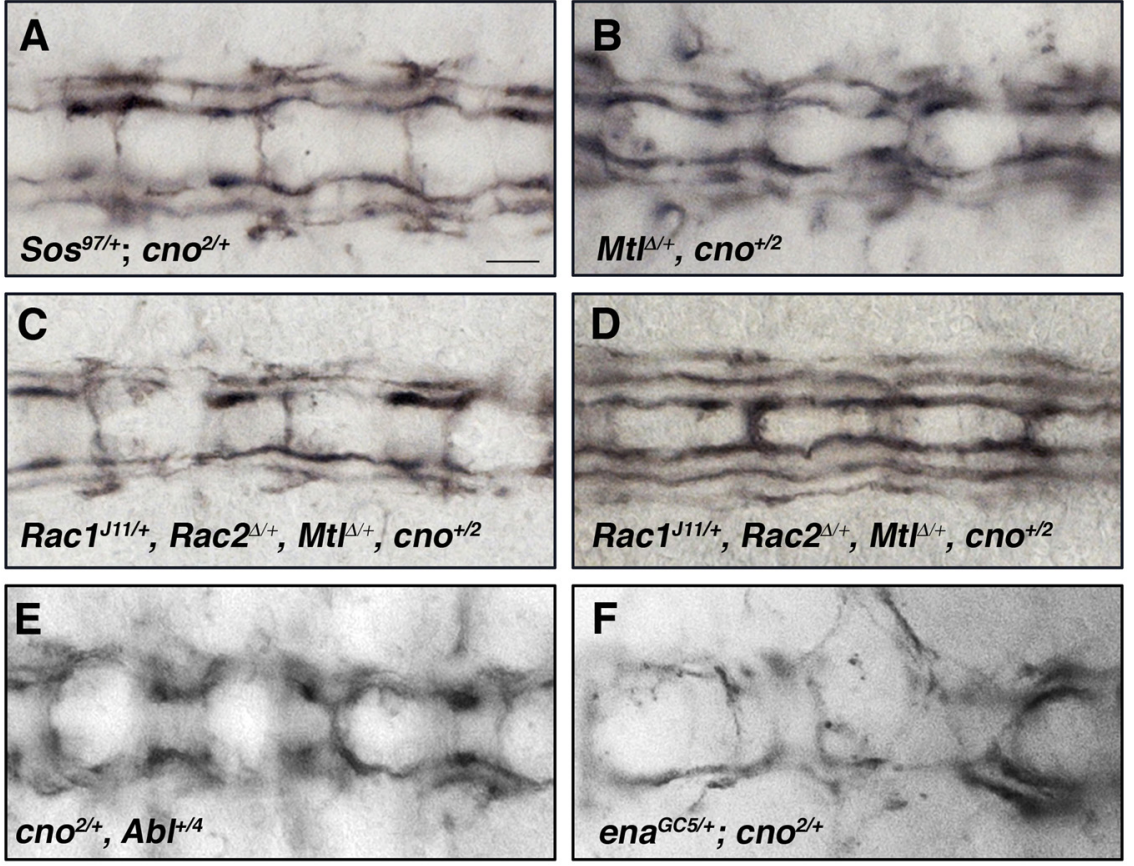

\begin{tabular}{|c|c|c|c|c|}
\hline \multicolumn{5}{|c|}{ Genetic interactions between $c n o$ and downstream components of the Slit/Robo pathway } \\
\hline \multirow[b]{2}{*}{ Genotype } & \multirow[b]{2}{*}{ \#embryos } & \multicolumn{3}{|c|}{ Axon pathfinding defects (\% embryos) } \\
\hline & & Total & Outgrowth & Guidance \\
\hline $\operatorname{Sos}^{34 E a-61+}$ & 41 & 0 & 0 & 0 \\
\hline $\mathrm{Cno}^{2 /+}$ & 40 & 0 & 0 & 0 \\
\hline $\operatorname{Sos}^{34 E a-61+} ; \operatorname{cno}^{2 /+}$ & 74 & $\begin{array}{l}14.9 \\
(11 / 74)\end{array}$ & $\begin{array}{c}91 \\
(10 / 11)\end{array}$ & $\begin{array}{l}63.6 \\
(7 / 11)\end{array}$ \\
\hline$M_{t l^{N+}}$ & 33 & 0 & 0 & 0 \\
\hline $\mathrm{cno}^{2 /+}, \mathrm{Mtl}^{/ 4}$ & 55 & $\begin{array}{l}14.5 \\
(8 / 55)\end{array}$ & $\begin{array}{l}100 \\
(8 / 8)\end{array}$ & $\begin{array}{l}12.5 \\
(1 / 8)\end{array}$ \\
\hline $\operatorname{Racl}^{I 11 /+}, \operatorname{Rac}^{\mathbf{\alpha}^{\alpha+}}, \mathrm{Mtl}^{\beta_{+}}$ & 50 & $\begin{array}{c}2 \\
(1 / 50)\end{array}$ & $\begin{array}{l}100 \\
(1 / 1)\end{array}$ & $\begin{array}{l}100 \\
(1 / 1)\end{array}$ \\
\hline $\operatorname{Racl}^{J 11 /+}, \operatorname{Rac2}^{\omega_{+}}, \mathrm{Mtl}^{\psi_{+}}, \mathrm{cno}^{+/ 2}$ & $+12 \quad 59$ & $\begin{array}{c}20.3 \\
(12 / 59)\end{array}$ & $\begin{array}{c}75 \\
(9 / 12)\end{array}$ & $\begin{array}{l}25 \\
(3 / 12)\end{array}$ \\
\hline$A b b^{1++}$ & 37 & 0 & 0 & 0 \\
\hline $\mathrm{Cno}^{2 /+}, \mathrm{Abl}^{+/ 4}$ & 42 & $\begin{array}{c}33 \\
(14 / 42)\end{array}$ & $\begin{array}{c}85.7 \\
(12 / 14)\end{array}$ & $\begin{array}{c}100 \\
(14 / 14)\end{array}$ \\
\hline$e n a^{G C S /+}$ & 42 & 0 & 0 & 0 \\
\hline $\boldsymbol{e n a}^{G C S /+} ; \boldsymbol{e n o}^{2 /+}$ & 62 & $\begin{array}{c}\mathbf{2 4 . 2} \\
(15 / 62)\end{array}$ & $\begin{array}{c}86.7 \\
(13 / 15)\end{array}$ & $\begin{array}{c}73.3 \\
(11 / 15)\end{array}$ \\
\hline
\end{tabular}

Figure 3. Cno functionally interacts with the Robo downstream effectors Abl, Ena, Sos, and Rac GTPases. $A-F$, Ventral views of stage 16 embryos stained with anti-Fas2. Transheterozygote mutant embryos for $\mathrm{CnO}^{2}$ and $\operatorname{Sos}^{97}(\boldsymbol{A}), \mathrm{Mt}^{\Delta}(\boldsymbol{B}), \operatorname{Rac}^{111}, \operatorname{Rac}^{\Delta}{ }^{\mathrm{B}}$ $M t t^{\Delta}(\boldsymbol{C}, \boldsymbol{D}),\left.A b\right|^{4}(\boldsymbol{E})$, and ena ${ }^{G C 5}(\boldsymbol{F})$ showed specific guidance defects compared with WT embryos (see Fig. 1G). $\boldsymbol{G}$, Quantitations of guidance defects in the genotypes specified. Scale bar, $10 \mu \mathrm{m}$.

$1 G)$. In $c n o^{2 / 2}$ mutants, $58.3 \%$ of the mutant embryos analyzed $(n=120)$ showed defects in Fas2-positive axons, such as defasciculation $(45 \%)$, axon stall $(4.2 \%)$, and axon misguidance $(32.5 \%)$ (Figs. $1 H, 2 G)$. These defects might reflect early failures in axon pathfinding. Indeed, at early stages (stage $12 / 2-13$ ), when Fas2 pioneer neurons are extending their axons in WT embryos, we already observed defects in axon guidance (20.6\%) in $\mathrm{cno}^{2 / 2}$ mutants $(n=34)$ (Fig. $1 I, J)$. The same phenotypes were found using independent mutant alleles of $c n o$. Thus, $c n o^{R 2 / R 2}$ as well as
Cno $2 / D f(3 R) E D 5147$ showed similar axon misguidance phenotypes in $43.2 \%(n=$ $44)$ and $48.2 \%(n=56)$, respectively, of the mutant embryos analyzed, evidence that supports the idea that Cno has a function in axon pathfinding. The fraction of severe defects in axon guidance (i.e., midline crossovers) detected in these later genotypes was $25 \%(11 / 44)$ in $c n o^{R 2 / R 2}$ and $12.5 \%(7 / 56)$ in $c n o^{2 / D f(3 R) E D 5147}$. To analyze in more detail which specific neuronal traits might be affected in cno mutants, we made use of primary neuron cultures. Whereas axon length was unaffected in $\mathrm{cno}^{2 / 2}$ mutant neurons, the number of filopodia was significantly increased compared with WT neurons (Fig. $1 K$ ), suggesting that Cno influences the actin cytoskeleton of growth cones and/or the signals that regulate the formation of filopodia. Notably, the loss of Robo leads to increased numbers of filopodia in vivo (Murray and Whitington, 1999).

Cno functionally interacts with the SlitRobo signaling pathway during midline axon guidance

Some of the phenotypes observed in $\mathrm{cno}^{2 / 2}$ mutant embryos and primary neurons were reminiscent of those shown by robo mutants. For example, $4.2 \%$ of $c n o^{2 / 2} \mathrm{mu}$ tants (and $25 \%$ of $\mathrm{cno}^{R 2 / R 2}$ mutants) showed "robo-like" ectopic midline crossing phenotypes (Fig. $1 H$ ). Thus, to test whether Cno functionally relates to SlitRobo signaling, we analyzed the $c n o^{2 / 2}$ mutant phenotype in a sensitized genetic mutant background in which the Slit pathway was impaired through loss of one gene copy of either robo or slit (i.e., in slit $^{2 /+}$ or in robo $^{2 /+}$ heterozygote backgrounds). slit $^{2 /+} ; \mathrm{cno}^{2 / 2}$ mutant embryos showed midline axon guidance phenotypes significantly enhanced compared with $\mathrm{cno}^{2 / 2}$ mutant phenotypes (Fig. $2 A, G$ ). Similar results were found in $\mathrm{robo}^{2 /+} ; \mathrm{Cno}^{2 / 2}$ mutant embryos (Fig. 2B,G). Moreover, slit ${ }^{2 /+}$; $\mathrm{Cno}^{2 /+}$ or $\mathrm{robo}^{2 /+}$; $\mathrm{Cno}^{2 /+}$ double heterozygotes showed specific and strong phenotypes in axon guidance, whereas the single heterozygotes $\left(\mathrm{slit}^{2 /+}, \mathrm{robo}^{2 /+}\right.$, and $\mathrm{cno}^{2 /+}$ ) showed much weaker or no defects (Fig. $2 C, D, G)$. A strong genetic interaction in ectopic midline crossing was also observed between $r o b o^{2}$ and the $c n o^{R 2}$ null allele or deficiency $D f(3 R) E D 5147$ that entirely removes cno $\left(32.5 \%\right.$ in $\mathrm{robo}^{2 /+} \mathrm{cno}^{R 2 /+}, n=40 ; 28.6 \%$ in $\left.\mathrm{robo}^{2 /+} \mathrm{cno}^{\mathrm{Df}(3 R) E D 5147 /+}, n=42\right)$. Furthermore, the genes encoding the two coreceptors of Robo, $N r x-I V$ and $S d c$, showed clear genetic interactions with cno (Fig. $2 E-G$ ).

The Rac-GEF Sos links Robo to Rac activation in the context of midline axon guidance (Fan et al., 2003; Yang and Bashaw, 2006). Given that Cno interacts with Rac in other systems (Takahashi et al., 1998) and that Cno is an actin-binding pro- 
tein (Sawyer et al., 2009), we wondered whether Cno was also genetically interacting with these downstream effectors of the Robo pathway. The analysis of transheterozygotes revealed a functional interaction between Cno and Sos during axon pathfinding (Fig. $3 A, G$ ). Moreover, genetic interactions were found between Cno and $\mathrm{Mtl}$, the Drosophila Rac gene known to be most relevant during axonal midline guidance (HakedaSuzuki et al., 2002; Ng et al., 2002) (Fig. 3 B, G). Transheterozygotes combining cno with all three Drosophila Rac genes $\left(\mathrm{Racl}^{111 /+}, \mathrm{Rac}^{\Delta /+}\right.$, and $\left.\mathrm{Mtl} \mathrm{l}^{\Delta /+}, \mathrm{Cno}^{+/ 2}\right)$ displayed an even higher percentage of axonal midline crossings (Fig. $3 C, D, G$ ) because the three Rac genes are partially redundant in this context (Ng et al., 2002). Finally, the tyrosine kinase Abl and the actin regulator Ena, which are required for Robo signaling (Bashaw et al., 2000; Hsouna et al., 2003), also displayed strong genetic interaction with $\mathrm{cno}\left(\mathrm{cno}^{+/ 2}, \mathrm{Abl}^{4 /+}\right.$, and ena $\mathrm{GC5/+}^{\mathrm{C}}$; $\mathrm{no}^{2 /+}$; Fig. $\left.3 E-G\right)$. In contrast, no interactions were found between Cno and the adaptor protein Dreadlocks (Dock), the p-21 activated kinase (Pak), or the actin regulator Chickadee (Chic), other downstream effectors of Robo. In conclusion, these results clearly indicate a specific functional interaction between $\mathrm{Cno}$ and the Slit-Robo signaling pathway.

\section{Longitudinal Robo-expressing axons cross the midline in cno $^{2 / 2}$ mutant embryos}

Next, we investigated potential molecular mechanisms underpinning the positive effects of Cno on Robo signaling. Important clues came from the observation of Robo-positive axons in $\mathrm{cno}^{2 / 2}$ mutants. In WT embryos at stage 16, Robo is detected in all longitudinal axons at high levels and in commissural axons at very low levels (Fig. $4 A$ ). However, in $21.9 \%$ of $\mathrm{cno}^{2 / 2}$ mutant embryos $(n=32)$, increased levels of Robo were detected in commissural axons (Fig. 4B). Double-immunofluorescence analyses in $\mathrm{cno}^{2 / 2}$ mutants for Robo and the longitudinal axon fascicle marker Fas2 revealed colocalization in axon fascicles crossing the midline (Fig. 4C,D”). Hence, longitudinal Robopositive neurons can cross the midline in $\mathrm{cno}^{2 / 2}$ mutants. Together, our results strongly suggest that Cno is required for the proper function of the Robo-repulsive activity at the midline.

\section{Cno forms a complex with Robo in vivo and stabilizes Robo on filopodia membranes in vitro}

The detection of Robo in commissures of cno mutant embryos suggested that Cno regulates the spatial localization of Robo within neurons. To test this possibility at the single neuron level, we analyzed primary neuron cultures. When staining WT neurons for Robo in the absence of detergent (to only see protein exposed on the neuronal surface), we noticed the presence of prominent patches of Robo on filopodia (Fig. $5 A$ ). In contrast, $\mathrm{cno}^{2 / 2}$ mutant primary neurons showed a significant decrease of these filopodial accumulations (Fig. 5A). Furthermore, Western blots of $\mathrm{Cno}^{2 / 2}$ mutant embryo extracts revealed a decrease in the overall amount of Robo compared with WT embryo extracts (Fig. 5B). We next assessed whether Cno was forming a complex with Robo in vivo and found that Cno and Robo coimmunoprecipitated from embryo extracts (Fig. 5C). Thus, Cno forms a complex with Robo in vivo and this complex seems to be required for Robo localization to filopodial surfaces.

The comm mutant phenotype is suppressed by cno in a dose-dependent manner

Comm is essential to downregulate Robo in the axons that cross the midline (contralateral neurons), by sorting it to endosomes

\section{Robo}
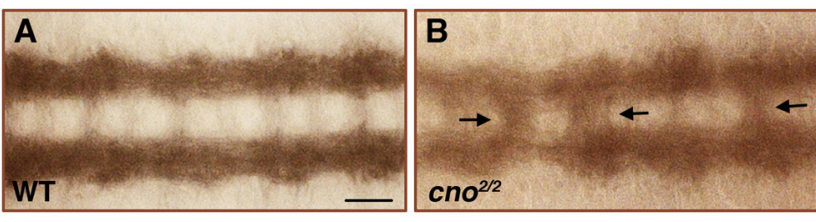

\section{Robo/Fas2}
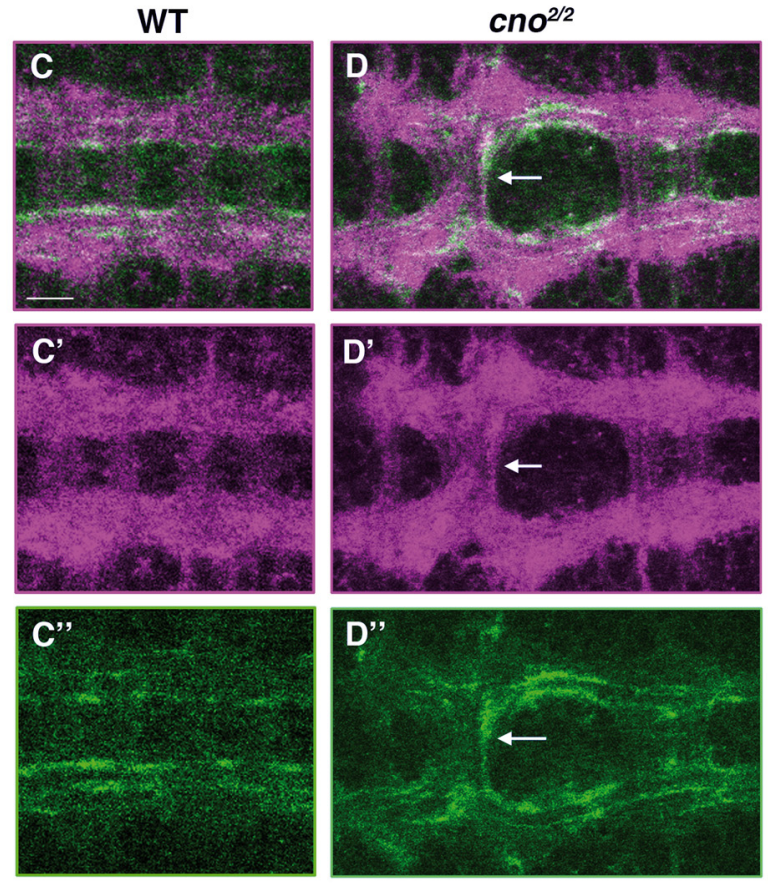

Figure 4. Longitudinal Robo/Fas2-expressing axons cross the midline in $\mathrm{cno}^{2 / 2}$ mutant embryos. $\boldsymbol{A}, \boldsymbol{B}$, Ventral views of stage 16 embryos stained with Robo. In WT embryos, Robo is detected at high levels along longitudinal tracts and at very low levels in commissural axons $(\boldsymbol{A})$. In $\mathrm{Cno}^{2 / 2}$ mutant embryos, Robo is distributed along longitudinal and commissural axons ( $\boldsymbol{B}$, arrows; see also text). C-D", Confocal micrographies of stage 16 embryos in a ventral view stained with Robo (magenta) and Fas2 (green). In WT embryos, longitudinal Fas2-expressing axons never cross the midline ( $\boldsymbol{C}\left(\boldsymbol{C}^{\prime \prime}\right)$. In $\boldsymbol{C n}^{2 / 2}$ mutant embryos, Robo/Fas2-positive axons are detected crossing the midline (D-D", arrows). Scale bar, $10 \mu \mathrm{m}$.

for degradation (Georgiou and Tear, 2002; Keleman et al., 2002, 2005). Accordingly, all axons in comm mutant embryos display active Robo and are repelled from the midline, so that commissures are completely absent (Tear et al., 1996) (Fig. 6B, compare with Fig. $6 A$ ). Based on our results, which strongly suggested that Cno is required for Robo signaling, we predicted that the comm mutant phenotype should at least partially depend on Cno. To test this hypothesis, we analyzed two different alleles of comm, the strong hypomorphic allele comm ${ }^{1}$ and the null allele $\mathrm{comm}^{5}$ (Tear et al., 1996), and combined them with the loss of function of cno. Analyses of these double-mutant embryos revealed that the lossof-commissure phenotype displayed by $\operatorname{comm}^{1 / 1}$ or $\mathrm{comm}^{5 / 5}$ was almost completely suppressed in a $\mathrm{cno}^{2 / 2}$ homozygous mutant background (Fig. 6D,E). Remarkably, the rescue of the comm phenotype by cno was dose-dependent, as in a $\mathrm{Cno}^{2 /+}$ heterozygote background the comm phenotype was already partially suppressed (Fig. 6C,E). This indicates that Cno levels are ratelimiting for midline repulsion, especially in the absence of Comm, when many more neurons display active Robo than in WT. These results, combined with our findings that Robo and Cno form a complex, led us to hypothesize that levels of Cno 

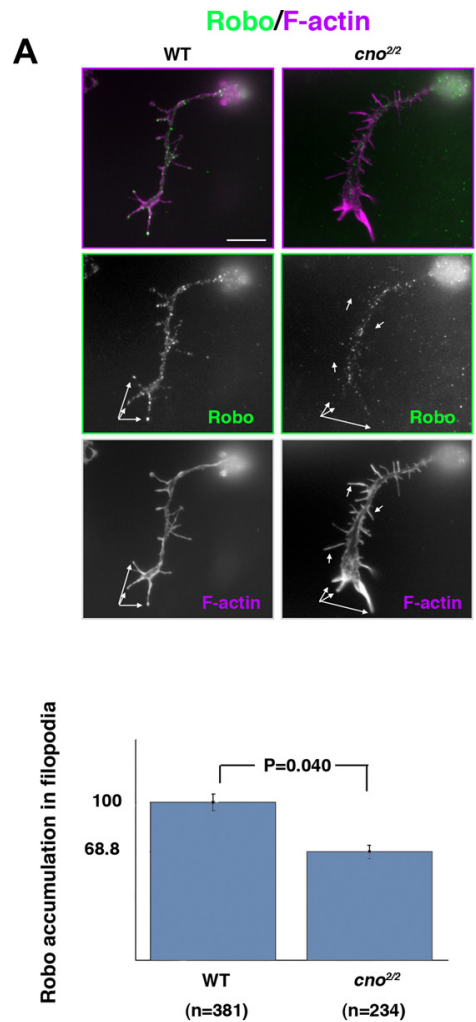

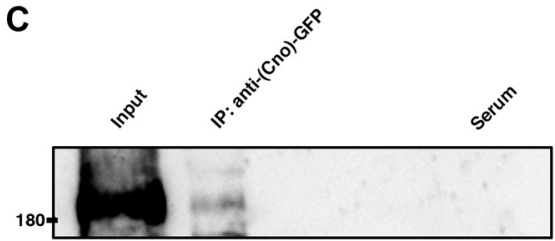

IB: anti-Robo

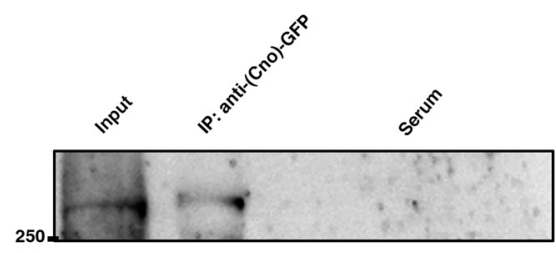

IB: anti-GFP
B
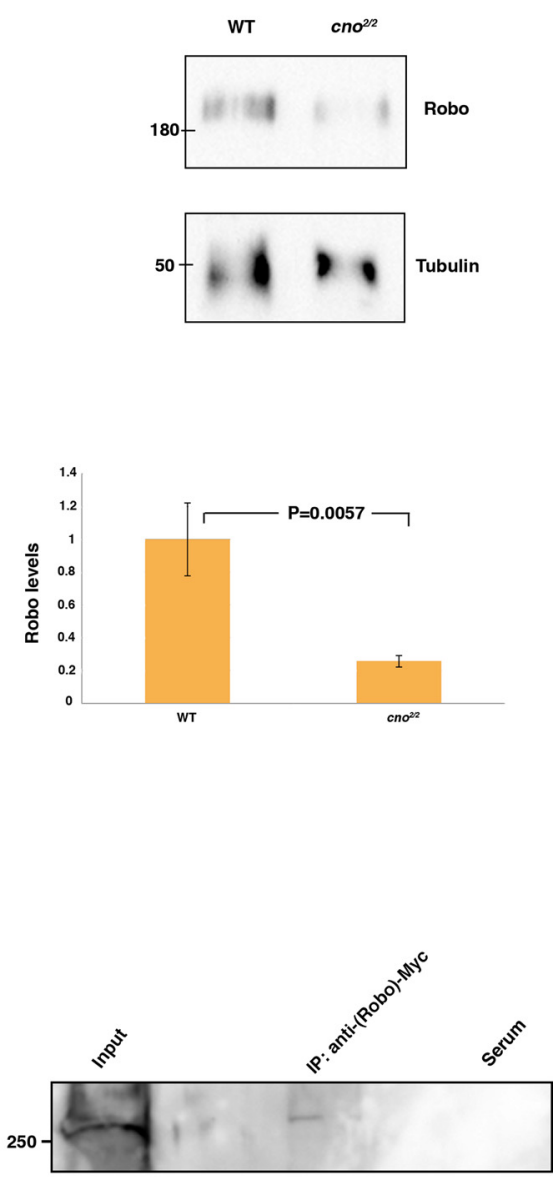

IB: anti-Cno

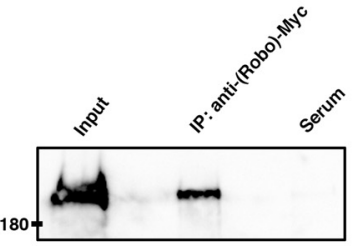

IB: anti-Myc

Figure 5. Cno forms a complex with Robo in vivo and regulates Robo subcellular localization in primary neurons. $\boldsymbol{A}$, Primary neurons stained with anti-Robo (green) and phalloidin (F-actin, magenta). In $c n 0^{2 / 2}$ mutant neurons, prominent Robo accumulations in filopodia (white arrows) are absent or decreased compared with WT neurons; quantifications are shown in the bar graph below ( $n$, number of neurons analyzed). In each neuron, Robo dots per filopodia were counted and then averaged and normalized with respect to the WT. $\boldsymbol{B}$, Western blots of $c \mathrm{CO}^{2 / 2}$ mutant embryo extracts revealed a decrease in the overall amounts of Robo compared with WT embryo extracts (tubulin was used as loading control). $C$, Lysates of embryos expressing (no-GFP or Robo-Myc were immunoprecipitated (IP) with rabbit anti-GFP or mouse antiMyc and probed on immunoblots (IB) with anti-Robo or with anti-Cno (upper blots) and with anti-GFP or anti-Myc (as IP controls, lower blots). In negative controls (Serum), embryo lysates were immunoprecipitated with unspecific rabbit or mouse serum and treated under the same conditions. Scale bar, $5 \mu \mathrm{m}$.

should be higher in comm mutant embryos than in their WT counterparts. Our Western blot analyses clearly confirmed this prediction, since Cno as well as Robo levels were more than doubled in $\mathrm{comm}^{5 / 5}$ mutant embryos (Fig. $6 \mathrm{~J}$ ).

Further studies in primary neurons showed that the functional relationships between Cno, Robo, and Comm observed in vivo apply to growth cones. First, comm ${ }^{1 / 1}$ mutant primary neurons displayed a significant increase in Robo accumulation in filopodial membranes compared with WT neurons (Fig. 6F-F', G-G',I), the opposite phenotype to that observed in $\mathrm{cno}^{2 / 2}$ mutant primary neurons (Fig. 6I, see also Fig. 5A). In $\mathrm{comm}^{1 / 1}, \mathrm{cno}^{2 / 2}$ double-mutant primary neurons, Robo localization in growth cone filopodia dropped significantly to WT levels (Fig. $\left.6 H-H^{\prime}, I\right)$. These results further reinforce the conclusion that Cno is an essential positive regulator of the Slit-Robo signaling pathway relevant for growth cone behavior.

\section{Discussion}

Slit and its receptor Robo are highly conserved and their function is key for mediating repulsive axon guidance at the midline, both in Drosophila and in vertebrates (Brose et al., 1999; Jen et al., 2004; Dickson and Gilestro, 2006; Ypsilanti et al., 2010). A tight regulation of this pathway during development is essential to ensure a correct final neural wiring and, hence, a functional nervous system. Many important aspects have been learned about the Robo pathway, but the intracellular mechanisms associated with Robo function remain poorly defined.

\section{Cno is a novel regulator of the Slit-Robo signaling pathway}

Here we present the scaffolding and actinbinding protein Cno as a novel positive regulator of the Slit-Robo signaling pathway. Cno functionally interacted with Nrx-IV and Sdc, other positive modulators of Robo activity that form a quaternary complex with Slit and Robo (Broadie et al., 2011). Additionally, cno mutant phenotypes were reminiscent of those described in Nrx-IV and $S d c$ mutants (Johnson et al., 2004; Steigemann et al., 2004; Chanana et al., 2009; Banerjee et al., 2010). For example, whereas in WT embryos Robo is only present at high levels in longitudinal connectives, in both cno and Nrx-IV mutants Robo was detected along with Fas2 fascicles crossing the midline. In WT embryos at later stages (stage16-17), Fas2 is present in three axon bundles at each side of the midline and the most medial (closer to the midline and known as the pCC/MP2 fascicle) includes ipsilateral neurons, such as pCC and dMP2 (Hidalgo and Brand, 1997). Thus, the presence of Fas2-positive axons crossing the midline in cno mutants strongly suggests that ipsilateral projecting axons in normal conditions are not properly repelled. Furthermore, our experiments in primary neurons clearly indicate that Cno is essential for Robo localization to filopodia, a typical location for receptors involved in axonal pathfinding. The upregulation of Cno in contralateral neurites at later stages, when they have already crossed the midline, might be related 


\section{BP102}
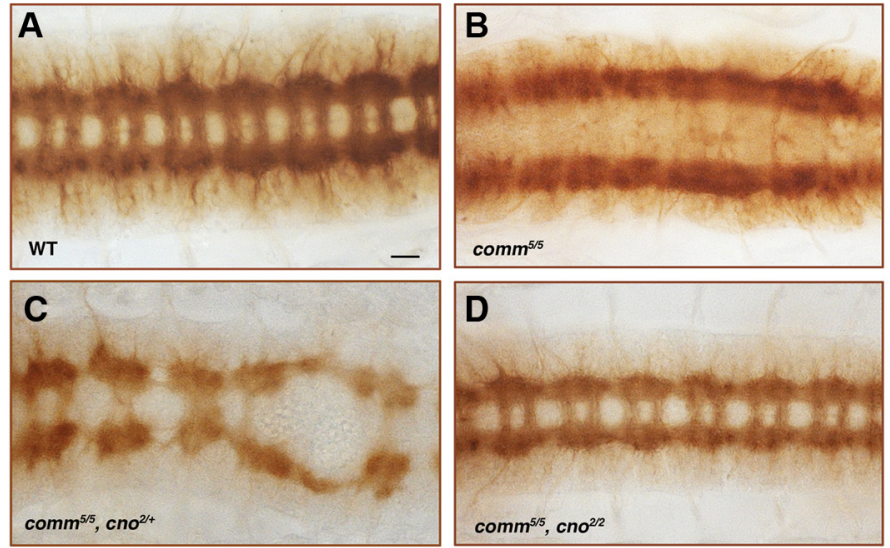

E

Commissure formation

\begin{tabular}{|c|c|c|c|c|}
\hline Genotype & $n *$ & a $(\%)$ & b (\%) & $\mathbf{a}+\mathbf{b}(\%)$ \\
\hline $\mathrm{CnO}^{2 / 2}$ & 444 & 1,1 & 0,0 & 1,1 \\
\hline $\mathrm{comm}^{1 / 1}$ & 482 & 88,0 & 4,8 & 92,7 \\
\hline $\mathrm{comm}^{5 / 5}$ & 120 & 100,0 & 0,0 & 100,0 \\
\hline $\mathrm{comm}^{1 / 5}$ & 208 & 96,2 & 1,4 & 97,6 \\
\hline $\mathrm{comm}^{1 / 1}, \mathrm{cno}^{2 / 2}$ & 605 & 3,6 & 10,3 & 13,9 \\
\hline $\mathrm{comm}^{5 / 5}, \mathrm{cno}^{2 / 2}$ & 903 & 3,0 & 10,7 & 13,7 \\
\hline $\mathrm{comm}^{1 / 5}, \mathrm{cno}^{2 / 2}$ & 216 & 8,8 & 7,4 & 16,2 \\
\hline $\mathrm{Cno}^{2 /+}$ & 45 & 0,0 & 0,0 & 0,0 \\
\hline $\mathrm{comm}^{1 / 1}, \mathrm{cno}^{2 /+}$ & 184 & 26,6 & 19,0 & 45,7 \\
\hline $\mathrm{comm}^{5 / 5}, \mathrm{cno}^{2 /+}$ & 261 & 34,9 & 16,1 & 51.0 \\
\hline
\end{tabular}

* segments scored

a commissures completely missin b commissures partially missing

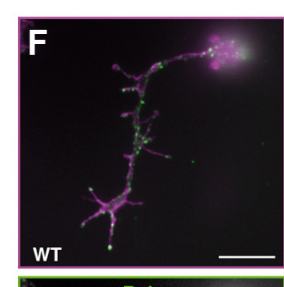

Robo/F-actin
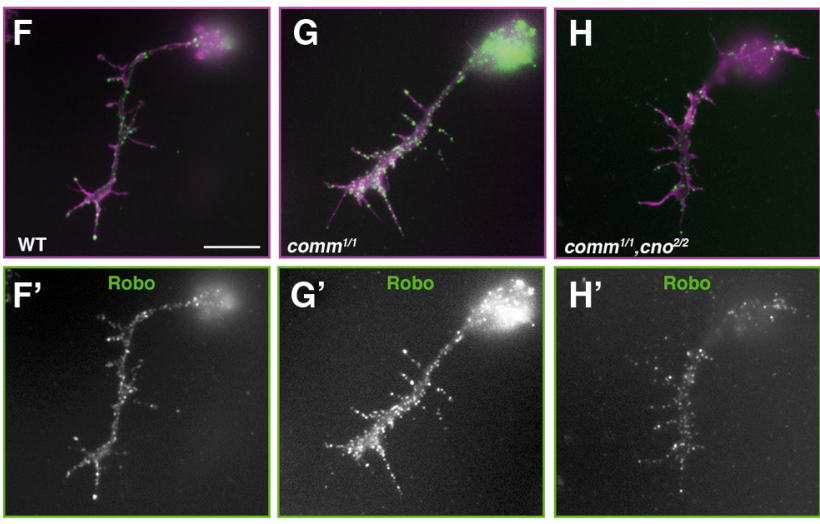

I

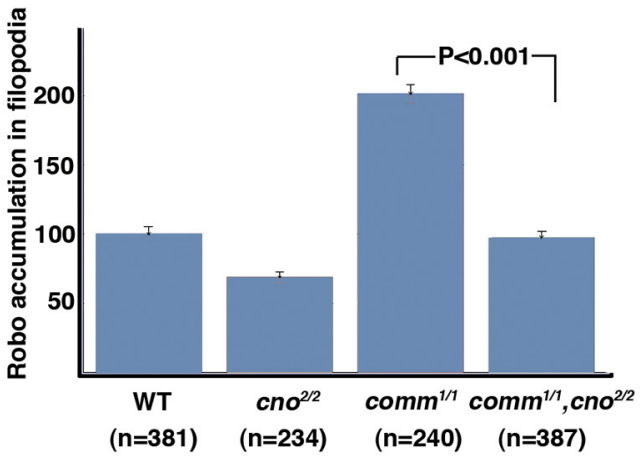

J
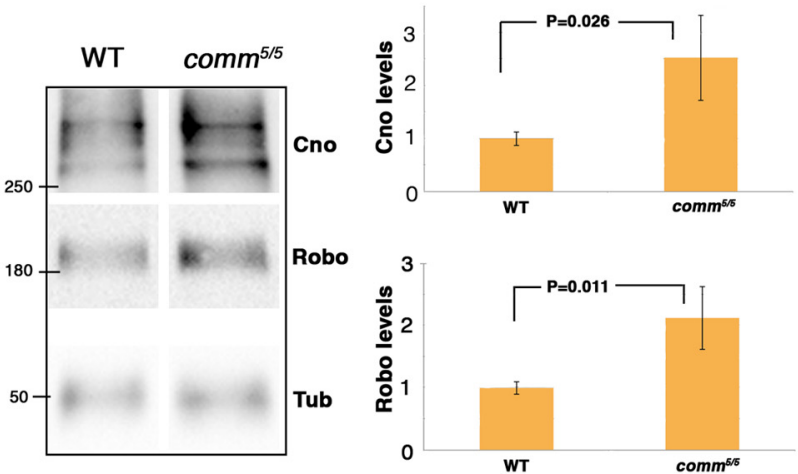

Figure 6. comm phenotype is suppressed by cno in a dose-dependent manner. $\boldsymbol{A}-\boldsymbol{D}$, Ventral views of stage 16 mutant embryos stained with mAb BP102 (a general CNS marker). In WT embryos, BP102 is detected along connectives and commissures $(\boldsymbol{A})$. No commissures are formed in comm $\mathrm{m}^{5 / 5}$ mutant embryos $(\boldsymbol{B})$. The comm $\mathrm{m}^{5 / 5}$ phenotype was suppressed in a cno ${ }^{2 / 2}$ homozygote mutant background (D). A partial suppression of the comm ${ }^{5 / 5}$ mutant phenotype was already observed by halving the dose of $\mathrm{cno}$ (C).E, Quantitations of commissure formation phenotypes in the genotypes specified. $\boldsymbol{F}-\boldsymbol{H}^{\prime}$, Primary neurons stained with Robo (green) and phalloidin (F-actin, magenta). In comm ${ }^{1 / 1}$ mutant neurons (G, G'), Robo accumulation at filopodia membranes increased compared with WT neurons $\left(\boldsymbol{F}, \boldsymbol{F}^{\prime}\right)$. In comm ${ }^{1 / 1}$, cno ${ }^{2 / 2}$ double-mutant neurons, Robo levels dropped to WT levels $\left(\boldsymbol{H}, \boldsymbol{H}^{\prime}\right)$. $\boldsymbol{I}$, Statistics of Robo accumulation in neuronal filopodia. Number of primary neurons analyzed $(n)$ is indicated below each of the genotypes specified. $J$, Western blots of comm ${ }^{5 / 5}$ mutant embryo extracts revealed an increase in the overall amount of both Robo and Cno compared with WT embryo extracts. Scale bar: (in $\boldsymbol{A}) \boldsymbol{A}-\boldsymbol{D}, 10 \mu \mathrm{m}$; (in $\boldsymbol{F}$ ) $\boldsymbol{F}-\boldsymbol{H}^{\prime}, 5 \mu \mathrm{m}$.

to the late function of Robo in preventing contralateral axons from recrossing or collapsing into the midline. Additionally, like Nrx-IV and Sdc, Cno was found in a complex with Robo in vivo. Intriguingly, both Nrx-IV and Sdc have potential PDZ-binding motifs at their $\mathrm{C}$ terminus specific for the PDZ domain of Cno (A. Carmena, unpublished observations). Moreover, Nrx-IV and Cno are able to coimmunoprecipitate in vivo (Slováková and Carmena, 2011). Then, Cno, by acting as a scaffold for these proteins could contribute to Robo stabilization at the membrane, a role analogous to that of the scaffolding protein $\beta$-spectrin (Garbe et al., 2007). However, the cytoplasmic part of both Nrx-IV and Sdc is not essential for Robo signaling (Johnson et al., 2004; Steigemann et al., 2004; Chanana et al.,
2009; Banerjee et al., 2010). Thus, Nrx-IV and Sdc are not the only link between Cno and Robo and additional proteins must be involved.

Remarkably, the loss of function of cno suppressed the phenotype of comm, which encodes a negative regulator of Robo in Drosophila (Tear et al., 1996; Georgiou and Tear, 2002; Keleman et al., 2002, 2005; Sabatier et al., 2004; Sánchez-Soriano et al., 2007). In comm mutants, Robo is upregulated in axons programmed to project contralaterally, thus aberrantly repelling them from the midline. Consequently, all neurons stay ipsilateral in comm mutants. Here, we observed that the levels of Cno are also upregulated in comm mutants. Moreover, the fact that cno suppressed the comm phenotype is strong proof that Cno is an important positive regulator of Robo function. 


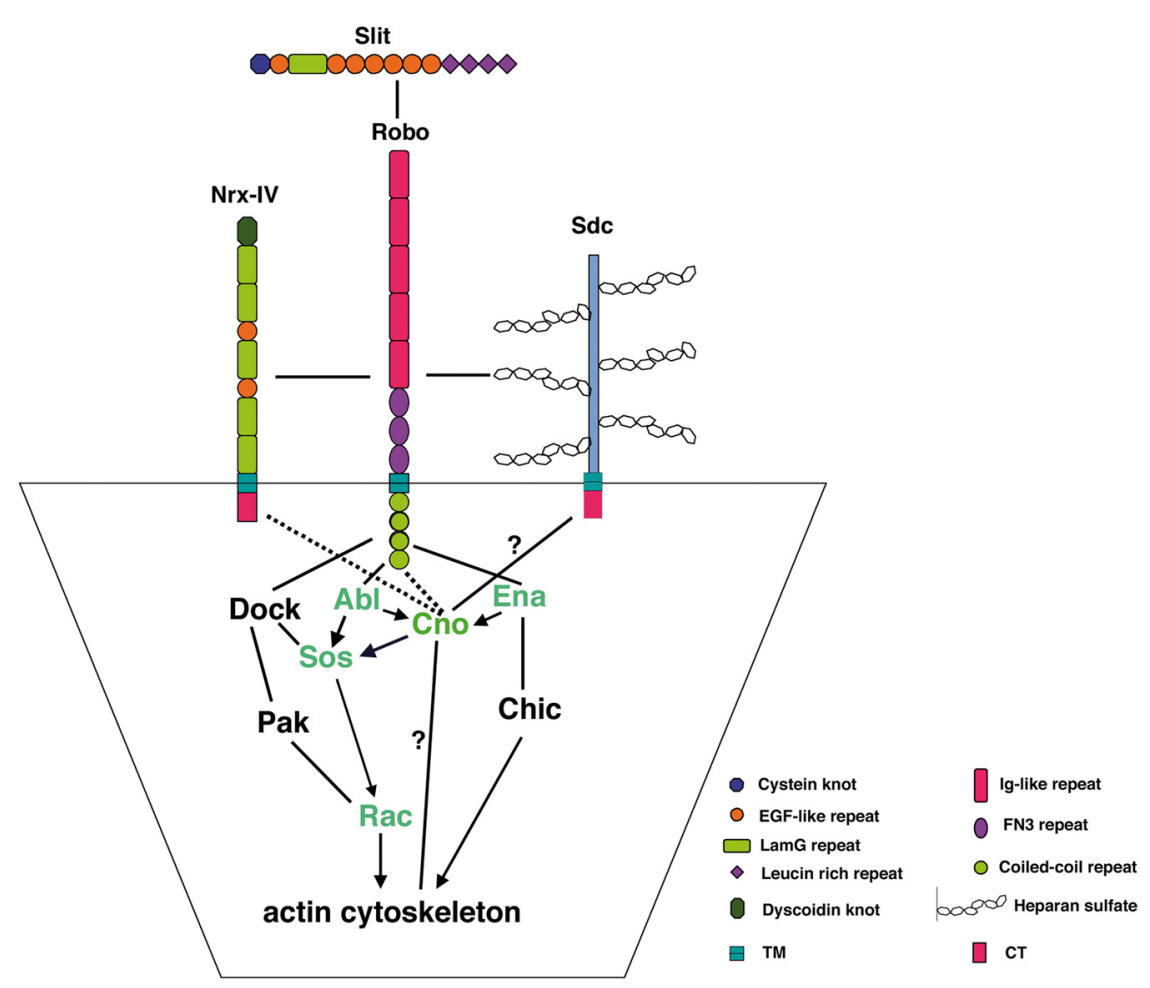

Figure 7. Cno is a novel regulator of the Slit-Robo signaling pathway. Slit-Robo signaling pathway during CNS midline axon pathfinding. Cno acts in a complex with Robo, contributing to Robo subcellular distribution at growth cones, and also functionally interacts with downstream effectors of Robo, such as Abl, Ena, Sos, and Rac GTPases, which in turn directly contribute to actin cytoskeleton rearrangements. Continuous lines indicate physical interactions; arrows indicate genetic, functional interactions; dotted lines between proteins indicate the presence of the proteins in a complex. Question marks denote potential physical interactions. LamG, laminin G; TM, transmembrane; FN3, fibronectin type 3; CT, C-terminal.

\section{Cno interacts with downstream components of the Robo signaling pathway}

Apart from genetically interacting with components of the quaternary Robo complex, Cno also functionally interacted with downstream components of the Robo pathway, such as Abl, the actin-regulator Ena, the Rac GTPases, and their GEF Sos, key transducers of Robo signaling (Bashaw et al., 2000; Hsouna et al., 2003; Yang and Bashaw, 2006; Broadie et al., 2011). Interestingly, cno also interacts genetically with Ena and Rac during the morphogenetic process known as the dorsal closure of the epidermis (Takahashi et al., 1998; Choi et al., 2011) and Cno has been shown to bind F-actin (Sawyer et al., 2009). In addition, Cno contains a Forkhead and a Dilute domain, both characteristic of proteins that interact with components of the cytoskeleton, microtubules and actin, respectively (Ponting, 1995). Hence, apart from acting as a member of the Robo complex that regulates its localization, Cno may also regulate Robo signaling at a more distal level of the receptor, impinging more directly on the actin cytoskeleton.

Together, our data unveil a novel function of Cno as a positive modulator of the Slit-Robo signaling pathway during CNS midline axon pathfinding (Fig. 7). Cno in a complex with Robo, and perhaps with other components of the pathway enriched at growth cones, stabilizes Robo at the membrane of specific subcellular locations. Cno also functionally interacts with the downstream components Abl, Sos, and Rac GTPases, which in turn modulate actin cytoskeleton rearrangements. The Slit-Robo pathway is very well conserved across species and members of the Robo pathway found first in Drosophila have been useful in the search for their vertebrate counterparts (Dickson and Gilestro,
2006; Ypsilanti et al., 2010). Given that the orthologues of Cno in vertebrates, AF-6 and Afadin, are also actin-binding proteins with a similar modular structure (Mandai et al., 1997; Lorger and Moelling, 2006), they might display a conserved function as regulators of the Slit-Robo pathway in mammals.

\section{References}

Banerjee S, Blauth K, Peters K, Rogers SL, Fanning AS, Bhat MA (2010) Drosophila Neurexin IV interacts with Roundabout and is required for repulsive midline axon guidance. J Neurosci 30:5653-5667.

Bashaw GJ, Klein R (2010) Signaling from axon guidance receptors. Cold Spring Harb Perspect Biol 2:a001941.

Bashaw GJ, Kidd T, Murray D, Pawson T, Goodman CS (2000) Repulsive axon guidance: Abelson and Enabled play opposing roles downstream of the Roundabout receptor. Cell 101:703-715.

Baumgartner S, Littleton JT, Broadie K, Bhat MA, Harbecke R, Lengyel JA, Chiquet-Ehrismann R, Prokop A, Bellen HJ (1996) A Drosophila neurexin is required for septate junction and blood-nerve barrier formation and function. Cell 87:1059-1068.

Broadie K, Baumgartner S, Prokop A (2011) Extracellular matrix and its receptors in Drosophila neural development. Dev Neurobiol 71:1102-1130.

Brose K, Bland KS, Wang KH, Arnott D, Henzel W, Goodman CS, Tessier-Lavigne M, Kidd T (1999) Slit proteins bind Robo receptors and have an evolutionarily conserved role in repulsive axon guidance. Cell 96:795-806.

Campos-Ortega JA, Hartenstein V (1997) The embryonic development of Drosophila melanogaster. Berlin: Springer.

Carmena A, Speicher S, Baylies M (2006) The PDZ protein Canoe/AF-6 links Ras-MAPK, Notch and Wingless/Wnt signaling pathways by directly interacting with Ras, Notch and Dishevelled. PLoS One 1:e66.

Chanana B, Steigemann P, Jäckle H, Vorbrüggen G (2009) Reception of Slit requires only the chondroitin-sulphate-modified extracellular domain of Syndecan at the target cell surface. Proc Natl Acad Sci USA 106:11984-11988.

Choi W, Jung KC, Nelson KS, Bhat MA, Beitel GJ, Peifer M, Fanning AS (2011) The single Drosophila ZO-1 protein Polychaetoid regulates embryonic morphogenesis in coordination with Canoe/afadin and Enabled. Mol Biol Cell 22:2010-2030.

Colamarino SA, Tessier-Lavigne M (1995) The role of the floor plate in axon guidance. Annu Rev Neurosci 18:497-529.

Dickson BJ, Gilestro GF (2006) Regulation of commissural axon pathfinding by slit and its Robo receptors. Annu Rev Cell Dev Biol 22:651-675.

Dittrich R, Bossing T, Gould AP, Technau GM, Urban J (1997) The differentiation of the serotonergic neurons in the Drosophila ventral nerve cord depends on the combined function of the zinc finger proteins Eagle and Huckebein. Development 124:2515-2525.

Evans TA, Bashaw GJ (2010) Axon guidance at the midline: of mice and flies. Curr Opin Neurobiol 20:79-85.

Fan X, Labrador JP, Hing H, Bashaw GJ (2003) Slit stimulation recruits Dock and Pak to the roundabout receptor and increases Rac activity to regulate axon repulsion at the CNS midline. Neuron 40:113-127.

Garbe DS, Das A, Dubreuil RR, Bashaw GJ (2007) beta-Spectrin functions independently of Ankyrin to regulate the establishment and maintenance of axon connections in the Drosophila embryonic CNS. Development 134:273-284.

Georgiou M, Tear G (2002) Commissureless is required both in commissural neurones and midline cells for axon guidance across the midline. Development 129:2947-2956. 
Gertler FB, Comer AR, Juang JL, Ahern SM, Clark MJ, Liebl EC, Hoffmann FM (1995) Enabled, a dosage-sensitive suppressor of mutations in the Drosophila Abl tyrosine kinase, encodes an Abl substrate with SH3 domain-binding properties. Genes Dev 9:521-533.

Grenningloh G, Rehm EJ, Goodman CS (1991) Genetic analysis of growth cone guidance in Drosophila: fasciclin II functions as a neuronal recognition molecule. Cell 67:45-57.

Hakeda-Suzuki S, Ng J, Tzu J, Dietzl G, Sun Y, Harms M, Nardine T, Luo L, Dickson BJ (2002) Rac function and regulation during Drosophila development. Nature 416:438-442.

Henkemeyer MJ, Bennett RL, Gertler FB, Hoffmann FM (1988) DNA sequence, structure, and tyrosine kinase activity of the Drosophila melanogaster Abelson proto-oncogene homolog. Mol Cell Biol 8:843-853.

Hidalgo A, Brand AH (1997) Targeted neuronal ablation: the role of pioneer neurons in guidance and fasciculation in the CNS of Drosophila. Development 124:3253-3262.

Hsouna A, Kim YS, VanBerkum MF (2003) Abelson tyrosine kinase is required to transduce midline repulsive cues. J Neurobiol 57:15-30.

Hummel T, Krukkert K, Roos J, Davis G, Klämbt C (2000) Drosophila Futsch/22C10 is a MAP1B-like protein required for dendritic and axonal development. Neuron 26:357-370.

Ito K, Urban J, Technau GM (1995) Distribution, classification and development of Drosophila glial cells in the late embryonic and early larval ventral nerve cord. Roux Arch Dev Biol 204:284-307.

Jen JC, Chan WM, Bosley TM, Wan J, Carr JR, Rüb U, Shattuck D, Salamon G, Kudo LC, Ou J, Lin DD, Salih MA, Kansu T, Al Dhalaan H, Al Zayed Z, MacDonald DB, Stigsby B, Plaitakis A, Dretakis EK, Gottlob I, et al. (2004) Mutations in a human ROBO gene disrupt hindbrain axon pathway crossing and morphogenesis. Science 304:1509-1513.

Johnson KG, Ghose A, Epstein E, Lincecum J, O'Connor MB, Van Vactor D (2004) Axonal heparan sulfate proteoglycans regulate the distribution and efficiency of the repellent slit during midline axon guidance. Curr Biol 14:499-504.

Jürgens G, Wieschaus E, Nüsslein-Volhard C, Kluding H (1984) Mutations affecting the pattern of the larval cuticle in Drosophila melanogaster. Roux Arch Dev Biol 193:283-295.

Keleman K, Rajagopalan S, Cleppien D, Teis D, Paiha K, Huber LA, Technau GM, Dickson BJ (2002) Comm sorts robo to control axon guidance at the Drosophila midline. Cell 110:415-427.

Keleman K, Ribeiro C, Dickson BJ (2005) Comm function in commissural axon guidance: cell-autonomous sorting of Robo in vivo. Nat Neurosci $8: 156-163$.

Kidd T, Brose K, Mitchell KJ, Fetter RD, Tessier-Lavigne M, Goodman CS, Tear G (1998) Roundabout controls axon crossing of the CNS midline and defines a novel subfamily of evolutionarily conserved guidance receptors. Cell 92:205-215.

Kidd T, Bland KS, Goodman CS (1999) Slit is the midline repellent for the robo receptor in Drosophila. Cell 96:785-794.

Klämbt C, Jacobs JR, Goodman CS (1991) The midline of the Drosophila central nervous system: a model for the genetic analysis of cell fate, cell migration, and growth cone guidance. Cell 64:801-815.

Lee T, Luo L (1999) Mosaic analysis with a repressible cell marker for studies of gene function in neuronal morphogenesis. Neuron 22:451-461.

Lorger M, Moelling K (2006) Regulation of epithelial wound closure and intercellular adhesion by interaction of AF6 with actin cytoskeleton. J Cell Sci 119:3385-3398.

Mandai K, Nakanishi H, Satoh A, Obaishi H, Wada M, Nishioka H, Itoh M, Mizoguchi A, Aoki T, Fujimoto T, Matsuda Y, Tsukita S, Takai Y (1997) Afadin: A novel actin filament-binding protein with one PDZ domain localized at cadherin-based cell-to-cell adherens junction. J Cell Biol 139:517-528.

Matsuo T, Takahashi K, Suzuki E, Yamamoto D (1999) The Canoe protein is necessary in adherens junctions for development of ommatidial architecture in the Drosophila compound eye. Cell Tissue Res 298:397-404.

Miyamoto H, Nihonmatsu I, Kondo S, Ueda R, Togashi S, Hirata K, Ikegami Y, Yamamoto D (1995) Canoe encodes a novel protein containing a GLGF/DHR motif and functions with Notch and scabrous in common developmental pathways in Drosophila. Genes Dev 9:612-625.
Morin X, Daneman R, Zavortink M, Chia W (2001) A protein trap strategy to detect GFP-tagged proteins expressed from their endogenous loci in Drosophila. Proc Natl Acad Sci U S A 98:15050-15055.

Murray MJ, Whitington PM (1999) Effects of roundabout on growth cone dynamics, filopodial length, and growth cone morphology at the midline and throughout the neuropile. J Neurosci 19:7901-7912.

Ng J, Nardine T, Harms M, Tzu J, Goldstein A, Sun Y, Dietzl G, Dickson BJ, Luo L (2002) Rac GTPases control axon growth, guidance and branching. Nature 416:442-447.

O'Donnell M, Chance RK, Bashaw GJ (2009) Axon growth and guidance: receptor regulation and signal transduction. Annu Rev Neurosci 32:383-412.

Ponting CP (1995) AF-6/cno: neither a kinesin nor a myosin, but a bit of both. Trends Biochem Sci 20:265-266.

Prokop A, Küppers-Munther B, Sánchez-Soriano N (2012) Using primary neuron cultures of Drosophila to analyze neuronal circuit formation and function. In: The making and un-making of neuronal circuits in Drosophila (Hassan BA, ed.). In press. New York: Humana.

Rogge RD, Karlovich CA, Banerjee U (1991) Genetic dissection of a neurodevelopmental pathway: Son of sevenless functions downstream of the sevenless and EGF receptor tyrosine kinases. Cell 64:39-48.

Rothberg JM, Hartley DA, Walther Z, Artavanis-Tsakonas S (1988) slit: an EGF-homologous locus of D. melanogaster involved in the development of the embryonic central nervous system. Cell 55:1047-1059.

Sabatier C, Plump AS, Le M, Brose K, Tamada A, Murakami F, Lee EY, Tessier-Lavigne M (2004) The divergent Robo family protein rig-1/ Robo3 is a negative regulator of slit responsiveness required for midline crossing by commissural axons. Cell 117:157-169.

Sánchez-Soriano N, Tear G, Whitington P, Prokop A (2007) Drosophila as a genetic and cellular model for studies on axonal growth. Neural Dev 2:9.

Sawyer JK, Harris NJ, Slep KC, Gaul U, Peifer M (2009) The Drosophila afadin homologue Canoe regulates linkage of the actin cytoskeleton to adherens junctions during apical constriction. J Cell Biol 186:57-73.

Schmid A, Chiba A, Doe CQ (1999) Clonal analysis of Drosophila embryonic neuroblasts: neural cell types, axon projections and muscle targets. Development 126:4653-4689.

Schneider I (1964) Differentiation of larval Drosophila eye-antennal discs in vitro. J Exp Zool 156:91-103.

Scholz H, Sadlowski E, Klaes A, Klämbt C (1997) Control of midline glia development in the embryonic Drosophila CNS. Mech Dev 64:137-151.

Seeger M, Tear G, Ferres-Marco D, Goodman CS (1993) Mutations affecting growth cone guidance in Drosophila: genes necessary for guidance toward or away from the midline. Neuron 10:409-426.

Slováková J, Carmena A (2011) Canoe functions at the CNS midline glia in a complex with Shotgun and Wrapper-Nrx-IV during neuron-glia interactions. Development 138:1563-1571.

Speicher S, Fischer A, Knoblich J, Carmena A (2008) The PDZ protein Canoe regulates the asymmetric division of Drosophila neuroblasts and muscle progenitors. Curr Biol 18:831-837.

Steigemann P, Molitor A, Fellert S, Jäckle H, Vorbrüggen G (2004) Heparan sulfate proteoglycan syndecan promotes axonal and myotube guidance by slit/robo signaling. Curr Biol 14:225-230.

Takahashi K, Matsuo T, Katsube T, Ueda R, Yamamoto D (1998) Direct binding between two PDZ domain proteins Canoe and ZO-1 and their roles in regulation of the jun $\mathrm{N}$-terminal kinase pathway in Drosophila morphogenesis. Mech Dev 78:97-111.

Tear G, Harris R, Sutaria S, Kilomanski K, Goodman CS, Seeger MA (1996) Commissureless controls growth cone guidance across the CNS midline in Drosophila and encodes a novel membrane protein. Neuron 16: 501-514.

Tepass U (1996) Crumbs, a component of the apical membrane, is required for zonula adherens formation in primary epithelia of Drosophila. Dev Biol 177:217-225.

Yang L, Bashaw GJ (2006) Son of sevenless directly links the Robo receptor to rac activation to control axon repulsion at the midline. Neuron 52:595-607.

Ypsilanti AR, Zagar Y, Chédotal A (2010) Moving away from the midline: new developments for Slit and Robo. Development 137:1939-1952. 